\title{
Parental School Involvement on Physical Activity and Screen Time Among Middle and High School Students
}

\author{
Kiran Thapa, $\mathrm{MPH}^{1}$, Joseph Charles Greer, $\mathrm{MPH}^{1}$, Jessica McNulty, $\mathrm{MPH}^{1}$, and Janani Rajbhandari-Thapa, $\mathrm{PhD}^{1}$ \\ ${ }^{1}$ Department of Health Policy and Management, College of Public Health, University of Georgia
}

Corresponding Author: Janani Rajbhandari-Thapa • College of Public Health, University of Georgia • 211 B Wright Hall (HSC), Athens, GA 30602 •

Telephone: (706) 713-2700•Email: jrthapa@uga.edu

\section{ABSTRACT}

Background: High levels of sedentary activity and low levels of physical activity have led to an increase in childhood obesity. A reduction of sedentary behavior and the promotion of an active lifestyle positively impacts weight status of children. A child may modify their behavior through direct interventions from their parents, such as enforcement of rules or guided activities, or through broad interventions, by expressing their values and modeling behavior. Current research does not establish if broad or specific parental involvement has a greater effect on healthy behavior. Our group sought to support existing evidence that parental involvement directly impacts behavior that reduces the likelihood of childhood obesity and determines if broad or specific parental involvement had a greater impact on healthy behavior.

Methods: The study population included all children grades 6-12 who completed the Georgia Student Health Survey 2.0. Survey questions from 674,354 students in Georgia were used for the analysis. Exploratory factor analysis (EFA) was conducted to derive a composite measure. Two factors were generated, broad parental involvement and specific parental involvement. Mixed-effects logistic regression was run using physical activity as the dependent variable and the two composite measures of parental involvement as predictors. The same mixed-effects logistic regression was performed using screen time as the dependent variable.

Results: Both measures of parental involvement showed a statistically significant positive relationship with physical activity. Both measures of parental involvement were significantly associated with screen time.

Conclusions: Results suggest that specific parental involvement is associated with an increase in weekly physical activity and a decrease in daily screen time. Increasing physical activity and reducing screen time have demonstrated clear reductions in the rate of obesity amongst children. To improve healthy behavior and reduce the likelihood of childhood obesity, parents should be directly involved in their child's school behavior.

Keywords: Obesity, childhood, parent involvement, screen time, school, physical activity.

\section{INTRODUCTION}

High levels of sedentary activity and low levels of physical activity have been implicated in an increase in childhood obesity (Jago et al., 2011). Childhood obesity is prevalent in the United States with $18.5 \%$ of children impacted by obesity (Anderson et al., 2019). Obesity patterns are set by a young age and childhood obesity is a strong predictor of adult obesity (Anderson et al., 2019). Obesity leads to high rates of morbidity and mortality in the U.S. (Anderson et al., 2019). Childhood interventions are important to obesity reduction efforts in the United States.

Research indicates reduction of sedentary behavior (by limiting screen time) and the promotion of an active lifestyle have a positive impact on the weight status of children (Decelis et al., 2014; Rhee, 2008). Additionally, sedentary time and screen time activities are found to be negatively associated with young people's physical health, health behaviors, and socio-cognitive outcomes (Amund et al., 2019). To reduce the incidence of obesity amongst school age children, practical interventions must be identified to encourage healthy behavior. By analyzing the relationship between physical activity, sedentary behavior, and screen time, research can identify the most effective interventions (Amund et al., 2019).

The role of parents' and parenting style in developing healthy behavior for their children has been well established (Snethen et al., 2016). Parenting style describes how a parent interacts and communicates with their child. Parenting style emphasizes the dispositional aspect of parenting or trait-like aspects (Langer et al., 2014). Parents may change their children's actions through role-modelling, parental practices and parental perceptions of their child's physical activity and sedentary behaviors (including screen time). A child may modify their behavior through direct interventions from their parents, such as enforcement of rules or guided activities, or through broad interventions, by expressing their values and modelling behavior ( $\mathrm{Xu}$ et al., 2015). 
Findings from recent studies and systematic reviews have demonstrated positive associations between parental involvement and physical activity and negative associations between parental involvement and screen time (Snethen et al., 2016). Interventions designed to reduce sedentary behavior have found family involvement as an important factor (Amund et al., 2019), however, these reviews have not established the amount of change in healthy behavior due to parental involvement. These reviews also did not establish if either broad or specific parental involvement had a greater effect on healthy behavior than the other. Our group sought to support existing evidence that parental involvement directly impacts behavior that reduces the likelihood of childhood obesity and determine if broad or specific parental involvement had a greater impact on healthy behavior.

\section{METHODS}

\section{Study Population}

The study population included all children who completed the Georgia Student Health Survey 2.0. The Georgia Student Health Survey 2.0 is a survey given to students in grades $6-$ 12 and was issued by the Georgia Department of Education to schools throughout the State of Georgia. Questions asked in the survey cover a variety of topics including assessment of school climate, parental involvement, and other factors concerning student health and well-being. Survey questions from 674,354 students in Georgia were collected and used for the analysis.

\section{Statistical Analysis}

Four 4-item Likert scale questions from the survey were determined to measure parental involvement. These questions were:

Question 46: My parents, or other adults at my home, think that education is important (Strongly disagree/ Somewhat disagree/Somewhat agree/Strongly agree).

Question 47: My parents, or other adults at my home, are able to help me with my homework when I ask them (Strongly disagree/Somewhat disagree/Somewhat agree/Strongly agree).

Question 48: My parents, or other adults in my home, ask me about my grades on a regular basis (Strongly disagree/Somewhat disagree/Somewhat agree/Strongly agree).

Question 49: My parents, or other adults at my home, think that it is important for me to graduate from high school (Strongly disagree / Somewhat disagree / Somewhat agree/Strongly agree).
Exploratory factor analysis (EFA) was conducted to derive a composite measure. Two factors were generated, broad parental involvement and specific parental involvement. Broad parental involvement is a composite of question 46 and question 49 and reflects student perception of the amount that their parents value their education and general success in school. Specific parental involvement is a composite of question 47 and question 48 and demonstrates the extent of direct parental involvement and intervention in a student's schoolwork.

Mixed effects logistic regression was run using physical activity as the dependent variable and the two composite measures of parental involvement as predictors. Physical activity was measured as number of days per week that the students reported exercising for greater than 60 minutes. Students were divided into two groups based on their level of activity: 4-5 days/week and $<4-5$ days/week with greater than 60 minutes of activity. The mixed-effects logistic regression model was adjusted for grade and gender.

The same mixed-effects logistic regression was performed using screen time as the dependent variable. Screen time was also divided into two groups: $>4-5$ hours/day and $<4-5$ hours/day of screen time.

\section{RESULTS}

\section{Effects of Parental Involvement on Physical Activity}

Both measures of parental involvement showed a statistically significant positive relationship with physical activity (Table 1). A one-unit Likert scale increase in specific parental involvement was associated with an expected increase of $36 \%$ in the odds of a child being physically active ( $>4-5$ days/week). Similarly, a one-unit Likert scale increase in broad parental involvement was associated with an expected increase of $4 \%$ in the odds of a child being physically active.

\section{Effects of Parental Involvement on Screen Time}

Both measures of parental involvement were also significantly associated with screen time (Table 2). A one-unit Likert scale decrease in specific parental involvement was associated with an expected decrease of $21 \%$ in the likelihood of a child experiencing excessive screen time ( $>4-5$ hours/day). A one-unit Likert scale increase in broad parental involvement is associated with a $22 \%$ increase in the likelihood of a child being an excessive screen-viewer. 
Table 1

Logistic regression results for physical activity

\begin{tabular}{lccc}
\hline & Estimate (OR) & Lower Limit & Upper Limit \\
\hline (Intercept) & $0.789^{* * *}$ & 0.765 & 0.813 \\
parent_invol_broad & $1.035^{* * *}$ & 1.028 & 1.042 \\
parent_invol_specific & $1.355^{* * *}$ & 1.346 & 1.364 \\
Grade 7 & $0.975^{* * *}$ & 0.957 & 0.993 \\
Grade 8 & $0.905^{* * *}$ & 0.889 & 0.922 \\
Grade 9 & $0.837^{* * *}$ & 0804 & 0.872 \\
Grade 10 & $0.701^{* * *}$ & 0.673 & 0.730 \\
Grade 11 & $0.651^{* * *}$ & 0.625 & 0.679 \\
Grade 12 & $0.624^{* * *}$ & 0.599 & 0.650 \\
Gender Male & $2.152^{* * *}$ & 2.130 & 2.173 \\
\hline
\end{tabular}

Note: $* * *$ is $\mathrm{p}, 0.001,{ }^{* *}$ is $\mathrm{p}<0.01$ and $*$ is $\mathrm{p}<0.05$.

Table 2

Logistic regression results for screen time

\begin{tabular}{lccc}
\hline & Estimate (OR) & Lower Limit & Upper Limit \\
\hline (Intercept) & $0.047^{* * *}$ & 0.039 & 0.056 \\
parent_invol_broad & $1.219^{* * *}$ & 1.208 & 1.230 \\
parent_invol_specific & $0.792^{* * *}$ & 0.785 & 0.798 \\
Grade 7 & $1.180^{* * *}$ & 1.153 & 1.208 \\
Grade 8 & $1.258^{* * *}$ & 1.229 & 1.288 \\
Grade 9 & 1.080 & 0.989 & 1.179 \\
Grade 10 & 1.041 & 0.953 & 1.137 \\
Grade 11 & $0.905^{*}$ & 0.828 & 0.988 \\
Grade 12 & $0.869^{* *}$ & 0.795 & 0.949 \\
Gender Male & $0.982^{* *}$ & 0.969 & 0.995 \\
\hline
\end{tabular}

Note: $* * *$ is $\mathrm{p}, 0.001, * *$ is $\mathrm{p}<0.01$ and $*$ is $\mathrm{p}<0.05$.

\section{DISCUSSION}

By performing statistical analysis on questions taken from the Georgia Student Health Survey 2.0, this study generally reinforces existing evidence that parental involvement leads to an increase in physical activity and a decrease in daily screen time.
Both broad parental involvement and specific parental involvement was associated with statistically significant increases in the odds of a child participating in physical activity for at least 4 days per week. The impact of specific parental involvement was substantially greater than broad parental involvement, suggesting that behavioral changes from children are more likely to occur when their parents ask questions related to their school performance and 
participate in study activities with their children. The smaller impact of broad parental involvement on physical activity indicates that a child's perception of the amount that their parents value their schoolwork is less impactful than receiving direct assistance from their parents.

Broad parental involvement and specific parental involvement also impacted the amount of screen time a child engaged in. Specific parental involvement was associated with decreased odds of a child being an excessive screen viewer. This result was expected and is consistent with past evidence (Schmidt et al., 2012). As a parent is more engaged in a child's schoolwork, a child is less likely to spend a significant amount of time in front of screens. Broad parental involvement was associated with an increased odds of excessive screen watching. This result was unexpected but indicates that parent perception and value signaling is not sufficient in reducing screen time for children.

Both measures of involvement were based on the amount that a parent was involved in a child's schoolwork. The Georgia Health Student Survey 2.0 did not ask children to respond to questions related to parent involvement in a child's physical activity or screen time. As a result, all conclusions are based specifically on the impact that parental involvement in educational success has on physical activity or screen time.

\section{CONCLUSION}

Among students between grades 6 and 12, some parental influences were associated with a change in physical activity and screen time. Results from student surveys suggest that specific parental involvement had a positive relationship with weekly physical activity and a negative relationship with daily screen time. However, broad parental involvement did not lead to a substantial increase in student activity and was associated with an increase in daily screen time. Increasing physical activity and reducing screen time have demonstrated clear reductions in the rate of obesity amongst children. To improve healthy behavior and reduce the likelihood of childhood obesity, parents should be directly involved in their child's school behavior.

Since both measures were drawn from student surveys and asked questions about parental involvement in school activity, this study suggests that the amount a parent values and participates in their child's schoolwork has broader effects on seemingly unrelated healthy behaviors. This may be due to the likelihood of parents with high involvement in school also having high involvement in physical activity and screen time. Future studies should assess the direct relationships between parental involvement in screen time and activity and their impact on child behavior.

\section{Acknowledgements}

Funding for the study was provided by the Office of the President, UGA Presidents' Interdisciplinary Seed Grant.

\section{References}

Amund, L., Otto, R. F. S., Bente, W., Oddrun, S., \& Ellen, M. H. (2019). Associations between Family Structure and Young People's Physical Activity and Screen Time Behaviors. BMC Public Health, 19(1), 1-10. Doi:10.1186/s12889-019-6740-2

Anderson, P. M., Butcher, K. F., \& Schanzenbach, D. W. (2019). Understanding Recent Trends in Childhood Obesity in the United States. Economics and Human Biology, 34, 16-25. Doi:10.1016/j.ehb.2019.02.002

Decelis, A., Jago, R., \& Fox, K. R. (2014). Physical Activity, Screen Time and Obesity Status in a Nationally Representative Sample of Maltese Youth with International Comparisons. BMC Public Health, 14(1), 664. Doi:10.1186/1471-2458-14-664

Jago, R., Baranowski, T., Baranowski, J. C., Thompson, D., \& Greaves, K. (2005). BMI from 3-6 y of Age is predicted by TV Viewing and Physical Activity, Not Diet. International Journal of Obesity, 29(6), 557-564. Doi: 10.1038/sj.ijo.0802969

Langer, S. L., Crain, A. L., Senso, M. M., Levy, R. L., \& Sherwood, N. E. (2014). Predicting Child Physical Activity and Screen Time: Parental Support for Physical Activity and General Parenting Styles. Journal of Pediatric Psychology, 39(6), 633-642. Doi:10.1093/jpepsy/jsu021

Maddison, R., Mhurchu, C. N., Foley, L., Epstein, L., Jiang, Y., Tsai, M., Heke, I. (2011). Screen Time Weight-Loss Intervention Targeting Children at Home (SWITCH): A Randomized Controlled Trial Study Protocol. BMC Public Health, 11(1), 524. Retrieved from:

http:/www.biomedcentral.com/content/pdf/1471-2458-11-524.pd f

Rhee, K. (2008). Childhood Overweight and the Relationship between Parent Behaviors, Parenting Style, and Family Functioning. The ANNALS of the American Academy of Political and Social Science, 615, 11-37. Doi: $10.1177 / 0002716207308400$

Schmidt, M. E., Haines, J., O'brien, A., McDonald, J., Price, S., Sherry, B., \& Taveras, E. M. (2012). Systematic review of effective strategies for reducing screen time among young children. Obesity, 20(7), 1338-1354.

Snethen, J. A., Broome, M. E., Treisman, P., Castro, E., \& Kelber, S. T. (2016). Effective Weight Loss for Children: A Meta-Analysis of Intervention Studies 2002-2015. Worldviews on Evidence-Based Nursing, (4), 294-302.

Doi:10.1111/wvn.12156

Xu, H., Wen, L. M., \& Rissel, C. (2015). Associations of Parental Influences with Physical Activity and Screen Time among Young Children: A Systematic Review. Journal of Obesity, 2015, 1-23. Doi:10.1155/2015/546925

(C) Joseph Charles Greer, Kiran Thapa, Jessica McNulty, and Janani R. Thapa. Originally published in jGPHA (http://www.gapha.org/igpha/)

May 15, 2021. This is an open-access article distributed under the terms of the Creative Commons Attribution Non-Commercial No-Derivatives License (http://creativecommons.org/licenses/by/4.0/), which permits unrestricted use, distribution, and reproduction in any medium, provided the original work ("first published in the Journal of the Georgia Public Health Association...") is properly cited with original URL and bibliographic citation information. The complete bibliographic information, a link to the original publication on http://www.gapha.jgpha.org/, as well as this copyright and license information must be included. 